\title{
Variability of Frontal Alpha Wave in Response to Mood Induction via Visual Stimulus: A Quantitative Electroencephalographic Study
}

\author{
Kolsoom Rajabi, ${ }^{1}$ Mani Bahrami Monajemi, ${ }^{2}$ Sepehr Setareh, ${ }^{3}$ and Javad Setareh ${ }^{4,5,}$ \\ ${ }^{1}$ Sari Branch, Islamic Azad University, Sari, Iran \\ ${ }^{2}$ School of Psychology, University of Tehran, Tehran, Iran \\ ${ }^{3}$ School of Medicine, Shahid Beheshti University of Medical Sciences, Tehran, Iran \\ ${ }^{4}$ Psychiatry and Behavioral Sciences Research Center, Addiction Institute, Mazandaran University of Medical Sciences, Sari, Iran \\ ${ }^{5}$ Department of Psychiatry, School of Medicine, Mazandaran University of Medical Sciences, Sari, Iran \\ "Corresponding author: Javad Setareh, Assistant Professor of Psychiatry, Psychiatry and Behavioral Sciences Research Center, Addiction Institute, Mazandaran University of \\ Medical Sciences, Sari, Iran. Tel: +98-1133273024, Fax: +98-1133285109, E-mail: jsetareh@mazums.ac.ir
}

Received 2016 January 26; Revised 2016 October 10; Accepted 2016 November 10.

\begin{abstract}
Background: One of the prime areas in psychiatry is concerned with assessing emotions. Assessment of physiologic responses can be attained by various approaches. One of them is analyzing Electroencephalography (EEG).

Objectives: In current study, we aimed to assess Alpha wave in frontal region after inducing specific emotion by showing evocative video clips.

Materials and Methods: After a baseline in Eye Open situation recording, we showed 5 video clips that each induced specific emotions from five major ones (Neutral, Happiness, Sadness, Anger, Fear) to 66 healthy individuals, including 33 males and 33 females between 20 - 40 years old and EEG recording was continued simultaneously. Then, we analyzed their brain waves. Absolute power of Alpha wave Bands (Alpha 1 and Alpha 2) in frontal region (FZ, F4, F3) was analyzed via Wilcoxon test.

Results: With respect to eye open situation, all video clips made significant change in Alpha 2 in F3 and F4 ( $<<0.001)$ but not in Fz except for sadness. Sad inductive clip made a significant increase in Alpha 2 in Fz, also Alpha 1 in F3 and F4 (P $<0.001)$. Fz had no significant variability in all other emotions.

Conclusions: The most trenchant impact with respect to Alpha band was sadness, and the most exclusive finding in FZ was Alpha 2. Nonetheless, in two other zones (F3, F4), Alpha 1 was exclusive and Alpha 2 variability was non-exclusive. It appears plausible that sadness activates neuron groups, which are involved in generating Alpha waves.
\end{abstract}

Keywords: Alpha Rhythm, Electroencephalography, Emotions, Frontal Cortex

\section{Background}

Neuroscientists and scholars favor variability of brain waves induced by various emotions or emotional disorders (1). Webster dictionary describes Emotion as mental reaction experienced by boosted emotion, which is usually linked with specific subject and it is accompanied by behavioral/physiological variability (2). Six basic emotions (Sadness, Anger, Fear, Happiness, Surprise and Disgust), which earlier were discussed by Ekman, led to various studies in this area (1). Different emotions are linked with specific patterns from physiologic responses and probably the causes are rooted in Central Nervous System (CNS) rather than the external environment (3).

FMRI studies suggest that various regions of brains such as Anterior Cingulate Cortex (ACC), Amygdala and Prefrontal Cortex (PFC) play substantial role in assessing emotional stimulus and emotion regulation (4-7). It appears plausible that pathways or specific regions are closely linked with key elements of these emotions, which can be explored by implementing various imaging techniques
(6). Understanding and measuring emotional experiences have great importance regarding emotional assessment and various approaches have been implemented regarding physiological assessment of emotion (6).

Quantitative Electroencephalography is statistical assessment of EEG, which enables scholars to assess functional imaging of the brain (8). One of the advantages of QEEG in comparing to FMRI is maintaining timing accuracy, low price accessories and portability (9). QEEG have been used in various areas such as diagnosis of mood disorders, choosing drug and forecasting a prognosis and efficacy of a treatment (10-12).

Some studies have been conducted regarding assessing emotions via EEG, for instance categorizing emotions after audiovisual stimulation (9, 13-15). Assessing variability of brain waves under various circumstances such as mood variability due to sleep deprivation is one of the varied studies in this regard (16). Rest EEG is not solely enough for assessing emotional response capacity of individuals (17). By inducing emotions in a person and recording the 
brain function, electrophysiological variability can be determined. One of the methods is providing visual or auditory stimulus and subsequently recording brain's functional reaction $(15,18)$.

In current study with presenting instances of Video Clips (VC), we aimed to assess the induced variability in the brain waves. One of the gallant features of this study that differentiates it from studies in this area is that we used indigenous video in order to assess brain waves' variability. Furthermore, our study mainly focuse on absolute power of alpha rather than coherence, which have been studied mostly in other studies and dividing alpha wave into smaller range (Alpha 1, Alpha 2) provide more accuracy and efficiency in diagnosis and also therapeutic method such as neurofeedback $(7,14)$. According to recent literatures in mood disorders such as depressive disorders, alpha's malfunctioning mainly can be noticed in frontal and prefrontal regions. Furthermore, assessing Alpha wave in other regions (e.g., temporal region) are under influence of so many artifacts; hence, it is nearly impossible to assess alpha wave role without influences of other factors. Furthermore, strong evidence suggests that the role of frontal region in emotion regulation is undeniable $(7,15)$. The results of current study can be useful in clinical settings as well due to the role of impaired emotions in various disorders such as fear in panic disorders, anger in impulse control disorder and sadness in depression.

\section{Materials and Methods}

We provided sample by advertising and fliers. Examinee had minimum educational status of Diploma and age between 20 - 40. Exclusion Criteria: No history of mental illness in first and second grade family member; using drugs (Psychotropic drugs in recent 6 months and or other kind of drugs in recent month), History of head trauma, Stroke, Multiple sclerosis, head or brain related surgical operations, scar in head area, Headache, Migraine, Diabetes, Seizure, Cardiovascular surgery operation, Neck surgery, Visual/Auditory, History of Hypertension, History substance abuse, answering to GHQ (3) and having score more than 21. Finally, psychiatric interview was conducted by psychiatrist and if no problem was noticed, individual were included in study. Eventually, 66 (33 males, 33 females) were selected for this study.

\subsection{Inducing Emotions}

There is no Farsi Standardized clip with respect to inducing emotions; initially, we chose 25 short clips (4 minutes duration) in order to attain visual elements that induce neutral, sadness, happiness, anger and fear sepa- rately without inducing other emotions. Twenty candidates (10 men, 10 women) who maintained inclusion criteria of this study were selected as case group after signing a consent form. Subsequently, in a same setting that real test was about to happen; during 5 days 5 clips were showed to candidates. After watching a clip, they were asked to score the intensity of their experienced emotion (neutral, sadness, happiness, anger and fear) from 0 (minimum) to 10 (maximum). The clip, which maintained highest mean with respect to target emotion and the lowest mean for other emotions, considered as exclusive clip regarding target emotion.

- Neutral VC: Four minutes video, which was captured from a fitness training course taught by a trainer and it was confirmed after initial assessment (mean $=9.07$ and $\mathrm{SD}=$ 2.14). Mean and SD regarding other emotions were as mentioned below: Happiness (mean $=1.34$ and $\mathrm{SD}=0.94)$, Sadness (mean $=0.00$ and $S D=0.00)$, Fear (mean $=0.5$ and $S D$ $=0.47)$, Anger $($ mean $=0.51$ and $S D=0.73)$.

- Happy VC: Four minutes video was captured from a famous comic Persian soap opera series, which was confirmed after initial assessment ( mean $=8.5$ and $S D=2.06$ ). Mean and SD regarding other emotions were as mentioned below: Neutral (mean $=0.49$ and $S D=0.47$ ), Sadness (mean $=0.00$ and $\mathrm{SD}=0.00)$, Fear $($ mean $=0.00$ and $\mathrm{SD}=0.00)$, Anger $($ mean $=0.37$ and $S D=0.45)$.

-Sad VC: Four minutes video was captured from funeral of famous Iranian singer and playing his famous musical performance simultaneously, which was confirmed after initial assessment (mean $=9.13$ and $S D=2.16$ ). Mean and SD regarding other emotions were as mentioned below: Happiness (mean $=0.00$ and $S D=0.00)$, Neutral (mean $=$ 0.00 and $S D=0.00$ ), Fear (mean $=1.53$ and $S D=0.89$ ), Anger (mean $=0.69$ and $\mathrm{SD}=0.80$ ).

- Anger VC: Four minutes video, which had child abusing content and it was confirmed after initial assessment (mean $=9.44$ and $\mathrm{SD}=2.29$ ). Mean and SD regarding other emotions were as mentioned below: Happiness (mean $=$ 0.00 and $\mathrm{SD}=0.00)$, Neutral (mean $=0.00$ and $\mathrm{SD}=0.00$ ), Fear ( mean $=0.73$ and $S D=0.62$ ), and Sadness $($ mean $=2.64$ and $S D=1.04)$.

- Fear VC: Four minutes video was captured from a horror film named Annabelle (directed by John R.Leonetti and Written by Gary Duberman in 2014), which was confirmed after initial assessment (mean $=8.60$ and $S D=2.06$ ). Mean and SD regarding other emotions were as mentioned below: Happiness (mean $=0.00$ and $S D=0.00$ ), Sadness (mean $=0.49$ and SD $=0.47$ ), Neutral $($ mean $=0.44$ and SD $=0.57)$, Anger $($ mean $=0.44$ and $S D=0.66)$. 


\subsection{Experimental Procedures}

Firstly, the experiment was explained comprehensively to participants and after that consent forms were signed and obtained. While test was conducted, participants sat by 30 centimeters distance from computer desk. Handedness was controlled among participants because handedness will influence dominant hemisphere. Brain waves were recorded while participants were in a relaxing position, with their eyes open and while watching 5 inducing clips that evoked 5 different emotions which each of them lasted 4 minutes (neutral, sadness, happiness, anger and fear). Recording procedure was conducted by help of professional technician in a semi dark room, with a quiet atmosphere and controlled temperature between $22-24^{\circ}$ and in time period between $05.30 \mathrm{pm}$ to $07.30 \mathrm{pm}$. Interval between the video clips was 5 minutes and we allocated this time in order to minimize the influence of video clips on each other. Regarding women, test was conducted in a first monthly cycle and after menstruating period. We conducted this study in luteal phase of menstrual cycle because in second menstrual cycle there are unfavorable variations in brains waves due to factors such as premenstrual syndrome and premenstrual dysphoric disorder.

\subsection{Gathering QEEG Data}

For recording, Mitsar 201 amplifier and acquisition system with 21 electrodes via international 10/20 system and electro cap were used. Symbol rate 500 and impedance quality of $5 \mathrm{~K} \mathrm{ohm}$ were sustained. Low cut filter equal to 0.3 , high cut filter equal to 30 , and also 45 - $55 \mathrm{~Hz}$ filter were used. Initially, recording was conducted while participants were in relaxing state, with their eyes open and subsequently while they were exposed to five (4 minutes duration) emotional inducing video clips. Data were transferred to Neuroguide software and visual and motion artifacts were deleted manually and automatically via further revisions. Subsequently, absolute power of waves in range of 8 - $12 \mathrm{HZ}$, containing Alpha 1 (8-10HZ), Alpha 2 (10-12HZ) in FZ, F3 and F4 (Prefrontal) regions were assessed.

\subsection{Statistical Evaluation}

Data was analyzed via SPSS software, and Alpha absolute power $(1,2$, Total) was considered as dependent variable. With aid of K-S Kolmogorov-Smirnov test and with regard to value of $\mathrm{P}=0$, which is lower than conventional error (0.05) and consequently Zero hypothesis, that demonstrate normal distribution of all variables was rejected with respect to all variables. Furthermore, since hypothesis of study was assessing differences of two dependent variables, notifying nonparametric nature of variables, Wilcoxon test was used in order to assess the hypoth- esis of current study and Alpha 1 and 2 absolute power of 66 healthy individuals was assessed in FZ, F3 and F4 regions.

\section{Results}

Lower frequencies of Alpha were more sensitive to Sadness emotion in F3 and F4, but not other emotions. As frequency elevates, sensitivity regarding watching VC will be boosted as well and consequently, power variations among emotions will become meaningful but their exclusiveness will decrease in both electrodes.

Sadness emotion maintains highest impact on brain waves in Alpha 1 in F3 and F4. Furthermore, FZ electrode is linked more exclusively with this emotion, but in Alpha 2 and not Alpha 1. Right hemisphere dominancy of absolute power of alpha band and its subgroups were noticed rather than left hemisphere at rest (EO) in all participants during all video clips. No significant differences in absolute power were noticed between male and female before and during watching clips. Furthermore, Alpha 1 and Alpha 2 had similar amplitude changes in F3 and F4 during emotions induction (Table 1).

\section{Discussion}

In current study, we assessed electroencephalography characteristics induced by evocative video clip in prefrontal region; in this sense sad emotion had more effect on Alpha power bands in comparing to other emotions type, in all right and left frontal and also mid frontal regions. Although other regions such as temporal cortex were used for assessment of pleasant and unpleasant or neutral emotions but temporal region had high pollution of temporalis muscle artifact (19). There are various studies, which focus on frontal alpha activity and its relation to emotion and decrease of alpha activity induced by negative emotions (20). In some other studies, scholars focus on other disorders such as obsessive compulsive disorder (21) and mood disorders (22). Most of studies in this area focus on asymmetry, in this sense left frontal activity is consistent with negative emotion or mood disorders. However in this study we focused on (Fz, F3, F4) regions and alpha activity induced by specific emotions. Few studies have been conducted with respect to lower frequency versus higher frequency of alpha bands. The meaningful increase in alpha1 activity with respect to sad emotion can be related to emotion itself. Alpha2 changes induced by clips in F3 and $\mathrm{F} 4$ regions, can be partly related to non-emotional factors. Harmon-Jones found that alpha asymmetry can be linked to motivation and not to affective state (23). Furthermore, Denis and Solomon demonstrated that while person 
Table 1. No 1-Wilcoxon Test Results Regarding Alpha Absolute Power in Respond to Emotional Inducing Video Clips (VC)

\begin{tabular}{|c|c|c|c|c|c|c|}
\hline \multirow[t]{3}{*}{ Situation } & \multicolumn{6}{|c|}{ Electrode Bands } \\
\hline & \multicolumn{2}{|c|}{ F3 } & \multicolumn{2}{|c|}{ F4 } & \multicolumn{2}{|c|}{ FZ } \\
\hline & Alpha 1 & Alpha 2 & Alpha 1 & Alpha 2 & Alpha 1 & Alpha 2 \\
\hline \multicolumn{7}{|l|}{ Eyes Open } \\
\hline Mean & 3.83 & 2.93 & 4.50 & 3.31 & 4.80 & 3.37 \\
\hline SD & 2.275 & 1.518 & 3.189 & 1.814 & 3.139 & 1.669 \\
\hline \multicolumn{7}{|l|}{ Neutral VC } \\
\hline Mean & 4.31 & 4.12 & 4.91 & 4.54 & 5.09 & 4.47 \\
\hline SD & 2.523 & 2.614 & 3.170 & 3.131 & 3.371 & 3.137 \\
\hline Sig & 0.062 & 0.0000 & 0.068 & 0.000 & 0.086 & 0.212 \\
\hline \multicolumn{7}{|l|}{ HappyVC } \\
\hline Mean & 4.41 & 4.02 & 4.92 & 4.39 & 5.15 & 4.40 \\
\hline SD & 2.318 & 2.629 & 2.750 & 2.728 & 2.634 & 2.587 \\
\hline Sig & 0.072 & 0.0000 & 0.216 & 0.000 & 0.103 & 0.127 \\
\hline \multicolumn{7}{|l|}{ Sad VC } \\
\hline Mean & 5.42 & 4.72 & 5.90 & 5.02 & 6.40 & 5.42 \\
\hline SD & 3.462 & 3.602 & 3.772 & 3.671 & 4.563 & 4.774 \\
\hline Sig & 0.000 & 0.000 & 0.000 & 0.000 & 0.063 & 0.000 \\
\hline \multicolumn{7}{|l|}{ Anger VC } \\
\hline Mean & 4.52 & 4.25 & 4.81 & 4.44 & 5.14 & 4.57 \\
\hline SD & 2.680 & 2.793 & 2.801 & 2.826 & 2.730 & 2.618 \\
\hline Sig & 0.086 & 0.000 & 0.452 & 0.000 & 0.215 & 0.085 \\
\hline \multicolumn{7}{|l|}{ Fear VC } \\
\hline Mean & 4.76 & 4.44 & 5.01 & 4.68 & 5.01 & 4.44 \\
\hline SD & 3.584 & 3.709 & 3.569 & 3.654 & 2.788 & 2.803 \\
\hline Sig & 0.067 & 0.000 & 0.231 & 0.001 & 0.198 & 0.218 \\
\hline
\end{tabular}

is exposed to evocative video clips, environmental effects of attention and inducing emotions are reduced and video clip maintains prime impact (15). Also many other studies linked asymmetry to mood disorders and their symptoms $(20,24)$. However, it was shown that frontal area maintain effective role regarding emotion regulation via its interaction with amygdale (25) and also limbic system (26). In our study Alpha 2 activity increased meaningfully with response to sad emotion more specifically in $\mathrm{Fz}$ (mid frontal zone) there are few studies conducted with respect to $\mathrm{Fz}$ area in the past (15). In Kops's study, electrophysiological responses like HRV (Heart Rate Variability) related to emotions were assessed (27). It has been demonstrated that in emotional disorders, alpha variability occurs in frontal regions. Hence, we assessed wider range of emotions alpha bands (Alpha 1, Alpha 2) in FZ, F3 and F4 zones of prefrontal region.
Our results show that neutral video clip with open eyes state in prefrontal region and various frequencies didn't have meaningful rise. None of other emotions demonstrated meaningful rise with neutral video clip. Although, Alpha 1 bilaterally showed no meaningful increase versus eye open condition, except for sad emotion. Furthermore, Alpha 2 bilaterally show variability for all emotions. In other sense, Alpha 2 variability is non-exclusive and Alpha1 variability is exclusive with respect to sad emotion in right and left prefrontal regions (brain electrical activity recorded by F4 and F3 electrodes). Mid frontal region (Fz) maintained lowest variability rate and in other word, it is more exclusive region. In this region, meaningful increase in Alpha 2 but not Alpha 1 is noticed only for sadness emotion. With respect to meaningful increase while watching neutral video clip; Alpha 2 in F3 and F4 zones, may be due to processing of audiovisual data non-exclusively and not 
necessarily because of emotions. Additionally, left hemisphere relation to positive emotion were not seen in this study in Alpha band; however, right frontal Alpha activity was related to negative emotion as stated by Balconi and Ferrari (28). Few studies have been conducted so far with respect to mid frontal Alpha 2, however in current study we specifically assessed sadness emotion and Alpha 2. In contraire to previous studies, in current study, we chose video clips precisely in order to convey exact needed emotion and we chose these movies with respect to local socio-cultural context. All in all, sad emotion induces most variability in prefrontal region. It appears plausible that brain's electrophysiological response to other emotions, occurs in other regions and other frequencies, hence it is vital to conduct other studies with respect to these enquiries.

Limitations of Study: In current study, we assessed young individuals and a study with a broader age range is advised. Secondly, we assessed women in luteal phase of their menstrual cycle and it would be more comprehensive if we conduct future studies in complete menstrual cycle. Finally, we excluded left-handed individuals in this study; in future studies, it is advisable to include left-handed individual as well.

\subsection{Conclusion}

Sadness induced by video clip maintains significant increase in absolute power of Alpha band specially in Alpha 2 in Fz and also Alpha 1 in F3 and F4 regions. Other emotions induced increased Alpha 2 in F3 and F4 non-specifically. Hence, Fz is a candidate region for investigation about sadness and also depressive disorders.

\section{Acknowledgments}

We would like to thank all individuals who participated in the study.

\section{Footnotes}

Authors' Contribution: Kolsoom Rajabi and Mani Bahrami Monajemi conceived and designed the evaluation and conducted the whole process of this study. Javad Setareh collected and interpreted the clinical data and drafted the manuscript. Sepehr Setareh and Mani Bahrami Monajemi participated in conducting statistical analyses. All authors read and approved the final manuscript.

Declaration of Interest: None declared.

Funding/Support: None declared.

\section{References}

1. LeDoux J. Rethinking the emotional brain. Neuron. 2012;73(4):653-76. doi: 10.1016/j.neuron.2012.02.004. [PubMed: 22365542].

2. Simple Definition of emotion Available from: www.merriamwebster.com/dictionary/emotion.

3. Lee YY, Hsieh S. Classifying different emotional states by means of EEG-based functional connectivity patterns. PLoS One. 2014;9(4):e95415. doi: 10.1371/journal.pone.0095415. [PubMed: 24743695].

4. Huppert FA. Psychological Well-being: Evidence Regarding its Causes and Consequences. Health Well-Being. 2009;1(2):137-64.

5. Wager TD, Kang J, Johnson TD, Nichols TE, Satpute AB, Barrett LF. A Bayesian model of category-specific emotional brain responses. PLoS Comput Biol. 2015;11(4):e1004066. doi: 10.1371/journal.pcbi.1004066. [PubMed: 25853490].

6. Zotev V, Yuan H, Misaki M, Phillips R, Young KD, Feldner MT, et al. Correlation between amygdala BOLD activity and frontal EEG asymmetry during real-time fMRI neurofeedback training in patients with depression. Neuroimage Clin. 2016;11:224-38. doi: 10.1016/j.nicl.2016.02.003. [PubMed: 26958462].

7. Marzbani H, Marateb HR, Mansourian M. Neurofeedback: A Comprehensive Review on System Design, Methodology and Clinical Applications. Basic Clin Neurosci. 2016;7(2):143-58. doi: 10.15412/J.BCN.03070208. [PubMed: 27303609].

8. Gevins A, Leong H, Smith ME, Le J, Du R. Mapping cognitive brain function with modern high-resolution electroencephalography. Trends in neurosciences. 1995;18(10):429-36.

9. Ashton H, Reid K, Marsh R, Johnson I, Alter K, Griffiths T. High frequency localised "hot spots" in temporal lobes of patients with intractable tinnitus: a quantitative electroencephalographic (QEEG) study. Neurosci Lett. 2007;426(1):23-8. doi: 10.1016/j.neulet.2007.08.034. [PubMed:17888572].

10. Demerdzieva A, Pop-Jordanova N. Alpha asymmetry in QEEG recordings in young patients with anxiety. Macedonian Academy of Sciences and Arts, Section of Biological and Medical Sciences. 2011;32(1):229-44.

11. Kopecek M, Tislerova B, Sos P, Bares M, Novak T, Krajca V, et al. QEEG changes during switch from depression to hypomania/mania: a case report. Neuro Endocrinol Lett. 2008;29(3):295-302. [PubMed: 18580843].

12. Morgan ML, Cook IA, Rapkin AJ, Leuchter AF. Neurophysiologic changes during estrogen augmentation in perimenopausal depression. Maturitas. 2007;56(1):54-60. doi: 10.1016/j.maturitas.2006.05.010. [PubMed: 16835012].

13. Dan'ko SG, Grachev LV, Boitsova Iu A, Solov'eva ML. [Statistically significant reproducible differences of pericranial muscles EMG in beta and gamma frequency ranges when different emotional and cognitive states are compared]. Fiziol Cheloveka. 2014;40(2):5-13. [PubMed: 25272701].

14. Coburn KL, Lauterbach EC, Boutros NN, Black KJ, Arciniegas DB, Coffey $\mathrm{CE}$. The value of quantitative electroencephalography in clinical psychiatry: a report by the Committee on Research of the American Neuropsychiatric Association. J Neuropsychiatry Clin Neurosci. 2006;18(4):460-500. doi: 10.1176/jnp.2006.18.4.460. [PubMed: 17135374].

15. Dennis TA, Solomon B. Frontal EEG and emotion regulation: electrocortical activity in response to emotional film clips is associated with reduced mood induction and attention interference effects. Biol Psychol. 2010;85(3):456-64. doi: 10.1016/j.biopsycho.2010.09.008. [PubMed: 20863872].

16. Ferreira C, Deslandes A, Moraes H, Cagy M, Basile LF, Piedade R, et al. The relation between EEG prefrontal asymmetry and subjective feelings of mood following 24 hours of sleep deprivation. Arq Neuropsiquiatr. 2006;64(2b):382-7. [PubMed:16917605]. 
17. Tolegenova AA, Kustubayeva AM, Matthews G. Trait Meta-Mood, gender and EEG response during emotion-regulation. Pers Individ Dif. 2014;65:75-80.

18. Tomarken AJ, Davidson RJ, Henriques JB. Resting frontal brain asymmetry predicts affective responses to films. J Pers Soc Psychol. 1990;59(4):791-801. [PubMed: 2254854].

19. Kortelainen J, Vayrynen E, Seppanen T. High-frequency electroencephalographic activity in left temporal area is associated with pleasant emotion induced by video clips. Comput Intell Neurosci. 2015;2015:762769. doi: 10.1155/2015/762769. [PubMed: 25883640].

20. Brzezicka A, Kaminski J, Kaminska OK, Wolynczyk-Gmaj D, Sedek G. Frontal EEG alpha band asymmetry as a predictor of reasoning deficiency in depressed people. Cognition \& emotion. 2016:1-11.

21. Smith EE, Zambrano-Vazquez L, Allen JJ. Patterns of alpha asymmetry in those with elevated worry, trait anxiety, and obsessive-compulsive symptoms: A test of the worry and avoidance models of alpha asymmetry. Neuropsychologia. 2016;85:118-26.

22. Tas C, Cebi M, Tan O, Hizli-Sayar G, Tarhan N, Brown EC. EEG power, cordance and coherence differences between unipolar and bipolar depression. Journal of affective disorders. 2015;172:184-90.

23. Harmon-Jones E. Early Career Award. Clarifying the emotive func- tions of asymmetrical frontal cortical activity. Psychophysiology. 2003;40(6):838-48.

24. Stewart JL, Coan JA, Towers DN, Allen JJ. Resting and task-elicited prefrontal EEG alpha asymmetry in depression: support for the capability model. Psychophysiology. 2014;51(5):446-55.

25. Banks SJ, Eddy KT, Angstadt M, Nathan PJ, Phan KL. Amygdala-frontal connectivity during emotion regulation. Social cognitive and affective neuroscience. 2007;2(4):303-12.

26. Mohlman J, Eldreth DA, Price RB, Staples AM, Hanson C. Prefrontallimbic connectivity during worry in older adults with generalized anxiety disorder. Aging \& mental health. 2015:1-13.

27. Kop WJ, Synowski SJ, Newell ME, Schmidt LA, Waldstein SR, Fox NA. Autonomic nervous system reactivity to positive and negative mood induction: the role of acute psychological responses and frontal electrocortical activity. Biol Psychol. 2011;86(3):230-8. doi: 10.1016/j.biopsycho.2010.12.003. [PubMed: 21182891].

28. Balconi M, Ferrari C. Subliminal and supraliminal processing of facial expression of emotions: brain oscillation in the left/right frontal area. Brain Sci. 2012;2(2):85-100. doi:10.3390/brainsci2020085. [PubMed: 24962767]. 